\title{
Foreword
}

\section{A life in Science: In memory of Jean-Pierre Badiali}

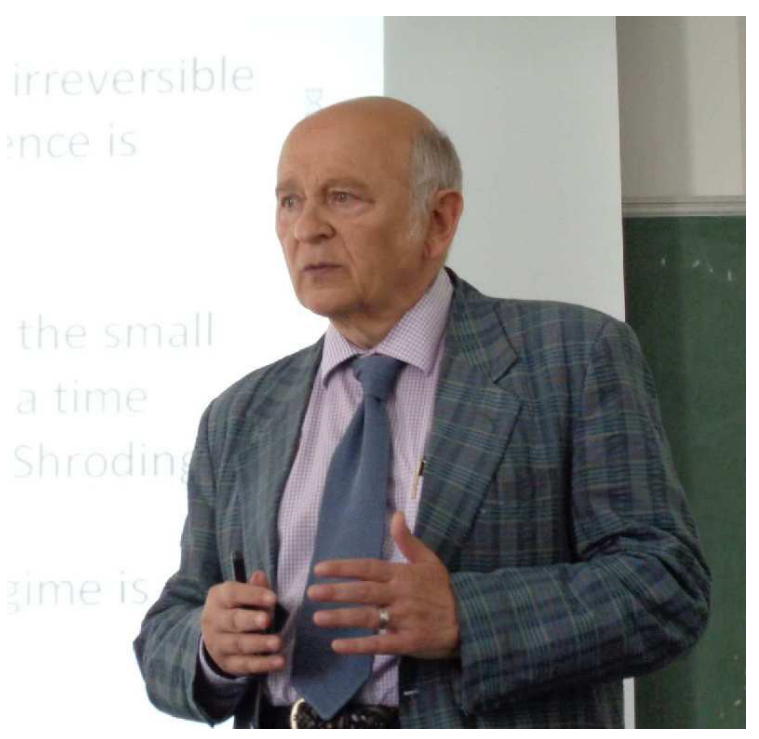

This issue is devoted to the memory of Jean-Pierre Badiali who passed away on April 15, 2016. His death is a great loss to all of us and to the whole scientific community. We express our deepest condolences to the family of Jean-Pierre Badiali, colleagues and friends. Jean-Pierre will remain in our memory as an outstanding scientist and an incredibly amiable person.

Jean-Pierre Badiali was born on October 20, 1941 in Mirande in the Midi-Pyrenées region in France. He graduated as an engineer from the Ecole Supérieure d'Electricité in 1965. He then entered the Centre National de la Recherche Scientifique (CNRS) as "Stagiaire de recherches". In 1969 he prepared a PhD “Contribution à l'étude des processus de relaxation électrique dans les solutions électrolytiques. Relations avec la solvatation et l'association ionique" 1 He worked in the CNRS research group "Physics of Liquids and Electrochemistry", directed by Prof. I. Epelboin. More specifically, he joined the team led by Dr. J.C. Lestrade, with the main objective to investigate the dielectric properties of electrolyte solutions. In 1967, he obtained a permanent position in the CNRS as a young scientist attaché de recherches. In 1980, he became Research Director in CNRS (at the time maître de recherches). He became the youngest Research Director in his laboratory.

In 1987, he received the "Silver Medal" of the CNRS for his contributions. And in the same year, he created the laboratory Structure and Reactivity at Interfaces (SRI) and directed it till 1991. The laboratory combined three teams. Two teams were more experimental, with focus on soft matter research subjects like wetting, Langmuir monolayers, micelles, colloids and electrochemistry. Jean-Pierre Badiali was in charge of the third team of theoreticians with different topics related to interfacial statistical physics, field theory. The challenge was to associate a theoretical and two experimental groups. These teams were directed by F. Brochard-Wyart, the second by M.P. Pileni. He has been four times elected as a member of the National Committee of the CNRS in the Section "Molecules: Structure and Interactions" and also president of this section between 1995 and 2000. He became First class research director in 1988. He then codirected the team "Energétique et Réactivité aux Interfaces" — Université Pierre et Marie Curie

${ }^{1}$ Contribution to the study of electrical relaxation processes in electrolitic solutions. Relations with solvation and ionic association 

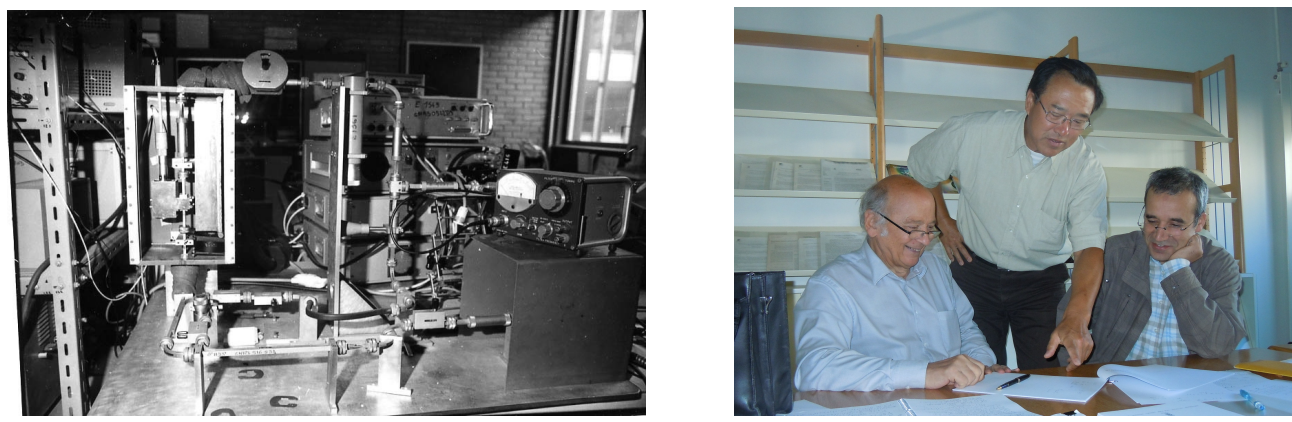

Figure 1. (Color online) Left: $35 \mathrm{GHz}$ interferometer in waveguide configuration developed by Jean-Pierre Badiali during his PhD. Right: A friendly image of Jean-Pierre sharing enthusiastic scientific discussion with his colleagues Q. Alexandre Wang (standing) and Aziz El Kaabouchi. We thank Hubert Cachet and Valentina Ivanova for providing these photographs.

with P. Letellier and then the team "Theory of Interfaces" in the "Laboratoire d'Electrochimie et Chimie Analytique" of the Ecole Nationale Supérieure de Chimie de Paris and Université P. et M. Curie. In 2001, he became research director of exceptional class. Jean-Pierre was also member of the Scientific council of the ISMANS22 engineer school from 1994 to 2010 and member of the board since 1996 until the end.

From his education, Jean-Pierre can be said to be an engineer in science. Due to his excellent scientific culture, communicative enthusiasm and insightful intuitions, Jean-Pierre worked in many different scientific domains including some very fundamental problems in quantum and statistical mechanics as well as some important applied research. When confronting a new field, Jean-Pierre first sought a broad view of the problem, reading relevant literature in order to propose some simple synthetic model which took into account the main features of the studied systems. He used to say that even a simple model could have a complex and rich behaviour. In scientific discussions, he was customary to introducing provocative questions which stimulated exchanges and would finally lead to a better understanding of the obtained results. In such a case, he would smile and said "Je suis Jean-provocateur".

Jean-Pierre Badiali started his research as an experimentalist ${ }^{3}$ His $\mathrm{PhD}$ was aimed at measuring the dielectric properties of electrolytes in different solvents and mixtures. Hubert Cachet gives a testimony of Jean-Pierre's results during this early period [1]. A contribution on solvent mixtures can be found in this issue [2]. We have found some documentation of this period (figure 1]. The different problems studied in electrochemistry, directed Jean-Pierre into developing statistical mechanical approaches for the description of the liquid state (liquids and also liquid metals) with a particular interest on systems with long range interactions (Coulombic, dipolar) and inhomogeneous systems (interfaces). However, we will see that his interests were much broader, we will see that they also include lithium batteries and corrosion problems. As mentioned in [1], developing a comprehensive study of the properties of the electrical interface, urged Jean-Pierre to also consider the metal contribution to the differential capacitance of the electrode/electrolyte systems. With coworkers he obtained important results for liquid metals (see [3], for example). Recent developments in the study of liquid metals are presented by two previous students of Jean-Pierre, Christian Régnaut and Saïd Amokrane [4]. In the 1970s, he spent several months in Syracuse University working with J. Goodisman. Together they rederived the fundamental Lippman equation, the Gibbs adsorption isotherm for an ideally polarisable electrode from the modern tools of statistical mechanics rather than from thermodynamics lifting some ambiguities and providing microscopic foundations [5-7]. An ideally polarisable electrode is shown to behave as a pure capacitance also under alternating current. Providing exact, deeper foundations to important relations is an example of the rigour of Jean-Pierre's scientific approach. Valuable results were then obtained for the ideally polarizable electrode including metal and solution contributions [8].

For another class of long range interactions, dipolar interactions, he then obtained fundamental results

\footnotetext{
${ }^{2}$ Institut supérieur des matériaux du Mans

${ }^{3}$ One of us recalls Jean-Pierre "boasting" with a smile that he could detect the failure of an electric engine from the shear sound, which is quite a feat for those who have known him in the continuation of his career!
} 
on dipolar liquids at interfaces. Studying density and orientation profiles at an interface [9-11], JeanPierre showed that a polar liquid at a planar interface is spontaneously birefringent as a consequence of the image potential which creates an alignment of the molecules leading to a spontaneous Kerr effect [12, 13] which can be observed in ellipsometry measurements. Some new results on dipolar systems are given in this issue by Dominique Levesque and by a former student of Jean-Pierre, Vincent Russier [14, 15].

The interest for long range interactions triggered the study of the long range correlations, typically investigating critical phenomena. Similar to his microscopic derivation of the Lipmann equations, JeanPierre was searching for an alternative description of the critical state avoiding the phenomenological Landau-Ginzburg Hamiltonian (LGH) based on symmetries to adopt quantities based directly on microscopies quantities [16, 17]. The so-called coupling constants of the critical field in the LGH are now integrals of the direct correlation functions. These integrals are related to thermodynamic quantities and are related to one another by the so-called Baxter relations which he discovered to be the equivalent of the Ward-Takahashi relations in field theory [18]. The study of the properties of the correlation functions at the critical point led him to rederive the basic Griffith-Kelly-Sherman correlation inequalities in the context of the liquid state [19].

The study of critical phenomena with the use of field theory prompted Jean-Pierre into investigating the possibility of a generic field theory framework for the liquid state to be applied, in particular, to ionic systems characterised by the long range Coulomb interactions. In the developed field theory approach similar to collective variables theory [20], the description of the system is given in the density fluctuation space, and the Hamiltonian of the system is presented as a sum of the interaction part and the ideal entropy part [21-24]. This approach was rather intuitive at the beginning but later the strong connection between field theory approach and collective variables theory was found [25, 26]. It was shown that the entropic term in the field theory is connected with the Jacobian of the transformation from the individual coordinates of particles to collective variables. The proposed expression for the entropic term in field theory is exact for the Jacobian in the case of point particles. The application of the field theory approach to inhomogeneous ionic fluids [27] shows that as a consequence of the entropic term, there is a strong coupling between the charge and the density fluctuations which leads to an ionic depletion at the neutral surface. This coupling is overlooked in well known approximations such as the Mean Spherical Approximation (MSA). Some other entropic effects for ionic systems are discussed in [28]. Comparison of the related Gaussian approaches and density functional theory approach (DFT) is also presented in this issue [29].

Jean-Pierre also discovered many other interesting phenomena in the field of electrochemistry. An intriguing result is the explanation of a special property of the electric differential capacitance. Experimentally, in the differential capacitance there was observed a portion of the curves that is independent of the nature of the ions. Jean-Pierre showed that this could be understood from a simple model based on a field theory, which is a generalization of the Gouy-Chapman theory taking into account specific interactions. In a mean field-like approach, the solution can be cast in a series of differential equations which have a one-parameter Lie group structure. This leads to predicting a desorption transition which explains that the capacitance curves for a given polarization are independent of the nature of the counterion, these having desorbed and no longer having specific interactions with the charged interface [30].

A new promising approach for the investigation of adsorption of fluids on crystalline surfaces was proposed by Jean-Pierre Badiali with coworkers in 1986 [31]. In this paper, the interface between a structural solid and a liquid is modelled by a flat surface with sticky sites placed on a regular lattice. After averaging over the fluid configurations, the model was mapped into a two-dimensional lattice-gas model with the interaction energies of the particles on the sites related to the mean force potential in an inhomogeneous fluid. For the simple case of a fluid of hard spheres, it was shown that the first layer of a fluid at contact with the solid can exhibit an order-disorder phase transition which can be interpreted as cooperative adsorption. Later this theory was generalized into associative fluids [32-334] where it was shown that due to the competition between intraparticle and interparticle correlations, a cooperative adsorption can appear at very small fluid densities. This phenomenon was used to explain an anomalous adsorption of polymers in the diluted-semidiluted concentration regime [35] and to clarify the mechanism of low-pressure insertion and separation of chain-like molecules in host matrices [36]. Together with coworkers Jean-Pierre generalized the approach to non-rigid surface. As a result, it became possible to investigate the modification of a surface by an adsorbent. It was shown that the presence of an adsorbent 
may induce distortive and roughening transitions on the underlaying substrate. A structural rearrangement of the surface and some other complex phenomena due to competing adsorbent-substrate interactions were observed [37-39]. Within the framework of this research, the effect of ice films morphology on $\mathrm{HCl}$ uptake was investigated as a possible mechanism of chlorine accumulation in polar stratospheric clouds [40, 41].

Another important aspect of these activities is connected with the investigations of the physics associated with intercalation of ions into host matrices. In these investigations, Jean-Pierre with coworkers extensively used the fact that an insertion process can be considered as a 3D adsorption. For the first time, they took into account the fact that a change of the volume of the host material may have a strong effect on the insertion mechanism. Employing the lattice gas model combined with the linear elasticity theory, they argued that major features of intercalation isotherms can be well understood in terms of the link between configurational and structural transitions [42-44]. The developed theory provides a quantitative description of different insertion processes and was used for the interpretation of the experiments in different experimental groups [45.47]. A natural extension of this research was connected with the investigation of the insertion of ions into a deformable host material. As a result, a link between electrical and mechanical properties was found. Such a link appears in the ion insertion processes, in the case of hydrogen sorption in pure metal or alloys as well as in the charging/discharging processes developed in polymers grafted on a metallic surface. This represents a new field of investigations associated with anomalous diffusion and possibly with non-standard thermodynamics.

Amongst theoretical works by Jean-Pierre in the theory of the electrical double layer, we also note a new exact relation for the charge profile or the so-called contact theorem [48, 49]. It establishes an exact expression for the contact value of the charge profile. The contact theorem for the density profile was known more than 30 years before [50, 51]. It is simple and local. The contact relation for the charge profile is far less intuitive and emphasizes the non-local character of the long range Coulomb interaction. The contact theorem for the charge profile triggered interesting papers and mediated scientific exchanges [52]. In his interest for charges at interfaces, Jean-Pierre also considered other aspects such as non-planar interfaces (modulated interfaces), or the presence of adsorbed species [53-55]. Different new results on the properties of the double layer are also presented in this issue [56-58].

Jean-Pierre also worked in close connection with experiments. For instance, for the description of the influence of proteins on some experimental features of inverse micelles embedding cytochrome-c, a simple model of associative sticky hard spheres was proposed [59, 60]. In the framework of this model, the stickness parameter and the size of hard spheres were found from small angle X-ray scattering (SAXS) measurements for empty micelles. The associative parameter was found from SAXS measurements for the micelles embedding protein. As a result, in the framework of the proposed model with one fitting parameter, it became possible to reproduce the main effects induced by the solubilization of cytochrome-c in reverse micelles, namely the change of the structure factor, the shift of the percolation threshold as well as the shift of the critical point for the liquid-liquid phase transition.

In the 90's, Jean-Pierre started working on lithium batteries and had ties and contracts with SAFT (Société des Accumulateurs Fixes et de Traction, company specialised in batteries) and the DRET (Direction des Recherches et Etudes Techniques). This work was very successful and opened up new directions in his research [62 64]. Starting from real problems connected with the properties of lithium batteries, he performed extensive simulations on the evolution of interfaces such as those that can be found in corrosion associated with metal dissolution or oxide growth. Due to numerous economical and environmental consequences, research in corrosion is a crucial subject which has been studied for centuries and owing to its complexity has been approached with several different viewpoints. Amongst its specificities, we note that it involves not only electrochemical anodic and cathodic reactions at the metal/solution interface. It spawns the paradoxical phenomenon of passivation-accelerating oxidation kinetics which may result in a decrease in the overall corrosion rate due to the formation of a protective layer, the so-called passive layer. With the experience gained from exploring the site poisoning effects in the study of lithium batteries, Jean-Pierre developed a mesoscopic description of corrosion based on a stochastic cellular automata (CA) description. In this framework, he was able to include the important characteristic features such as the diffusion of constituents, reactants and the spatial separation of anodic and cathodic reaction. His intuition was that, although the process at the local scale can be described by simple, elementary CA evolution rules, at a larger scale, collectively, there can be a subtle interplay 
between these phenomena and the overall morphology of the interfaces [61]. As a result, complex kinetics may emerge, the understanding of which is crucial in predicting the durability of the device. In this context, topological characterization of the interface is important. One can consider the roughness factor or the mean-square deviation of the front line from its mean position [65] and also what Jean-Pierre called the chemical roughness related to the number of reactive sites. Developed in two dimensions [66-73], these models are still being developed by collaborators and the recent results on corrosion are presented in this issue [74, 75] as well as complex disorder media, porous systems studies [76, 77]. Jean-Pierre considered corrosion as a method to produce new materials and worked in this spirit to see how it is possible to describe the formation of organized structures on a surface from the theoretical point of view [73]. This perspective is also presented in the issue [75] as well as in an experimental work [78].

Some of the recent works by Jean-Pierre and by his coworkers are related to the description of experimental facts related to the nuclear accident at Chornobyl (Ukraine). An example of this domain of investigation is presented in one of the last papers of Jean-Pierre published in this issue [79].

Works on lithium batteries also stimulated the interest of Jean-Pierre to fundamental problems related to the entropy of complex systems and non-extensive media. Statistical properties of such systems were studied within a combination of the maximum information principle and the super-statistical approach with parametrized information entropy measures such as Tsallis or Renyi [80-82]. This approach was developed for the investigation of the processes such as aging in lithium batteries.

During the last decades, Jean-Pierre Badiali worked a lot on many different fundamental problems of quantum and statistical physics. They concern the foundations of statistical mechanics via the path integral formalism and the Feynman conjecture, fractal behaviour of quantum systems [83, 84], the role of discreteness of space-time for quantum and statistical mechanics [85, 86]. The introduction of a quantized space-time shows that the thermodynamics is consistent with the equation of motion that is time-irreversible at microscopic level. As a result, the derivation of a $\mathrm{H}$-theorem becomes possible. The relation between action and entropy was illustrated in the case of black holes [87]. These researches open up the possibility of a new description of irreversible chemical reactions, the investigations of chemical reactions in small systems [88-91]. Different aspects and novelty of the last scientific works by Jean-Pierre are discussed in this issue by P. Riot and A. Le Méhauté [92].

In his scientific career, Jean-Pierre Badiali paid significant attention to working with young researchers and to collaborating with other scientific groups. He directed fourteen $\mathrm{PhD}$ thesis which included four Thèses d'Etat (old regime long research doctorates 5-7 years). Most of his students had prestigious careers, became professors of Universities, research directors in CNRS, engineers... . Due to a broad domain of activities, Jean-Pierre had many scientific collaborations with different groups in France as well as outside France. He had good collaboration with the Orsay group in computer simulations (Dominisque Levesque, Jean-Jacques Weiss, Jean-Michel Caillol), the Université d'Evry (Annie Chausse, Richard Messina, Christine Vautrin-Ul). During his scientific career, he visited many scientific centres in the world and had permanent scientific collaboration with them. Between them there is the Institute for Condensed Matter Physics (ICMP) of the National Academy of Sciences of Ukraine (NASU). A continuous and fruitful collaboration of Jean-Pierre Badiali with ICMP has been going on since 1991. He was an active participant of various joint international projects with ICMP. Jean-Pierre Badiali was a member of the Editorial Board of the "Condensed Matter Physics" journal. According to the decision of the Scientific Council of the ICMP of NAS of Ukraine from December 20, 2013 the title of Doctor honoris causa was conferred to Jean-Pierre Badiali for the basic results which provided explanation and description of various types of phenomena in electrochemistry, surface science and soft matter, for the development of new successful approaches in statistical mechanics as well as for his personal initiatives and participation in different projects aimed at strengthening collaboration between France and Ukraine. Jean-Pierre had also fruitful collaboration with Sasha Filipov from Physico-Technical Institute of NASU in Donetsk as well as very fruitful collaborations with the Institute of Physical Chemistry of the Polish Academy of Sciences (Janusz Stafiej, Zofia Borkowska), the Frumkin Institute for Electrochemistry of Russian Academy of Sciences (Misha Vorotyntsev and Sasha Kuznetsov), with different scientific centres in Germany (Frank Forstmann from Freie Universität Berlin, Wolfgang Schmickler from Ulm University), Puerto Rico University (Lesser Blum), Universidade Federal Fluminense in Brasil (Fabio Aarão Reis), Université Libre de Bruxelles (Claudine Buess-Herman and Mireille Poelman), and many others scientific centres. 


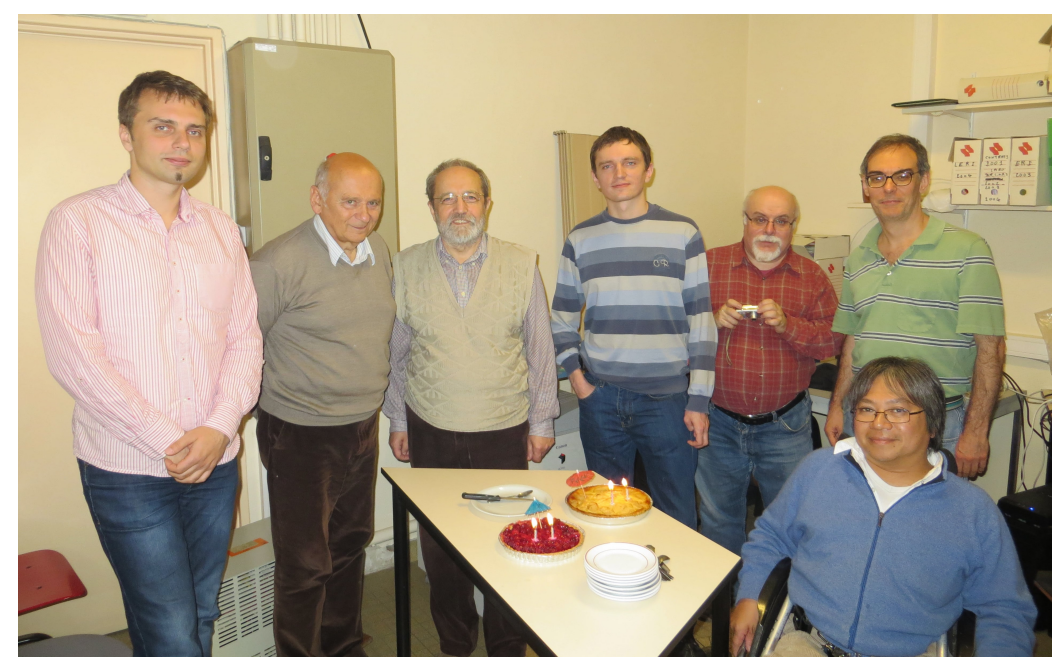

Figure 2. (Color online) The birthday of Jean-Pierre Badiali in his laboratory in 2013. From left to right: Taras Patsahan (Ukraine), Jean-Pierre Badiali, Myroslav Holovko (Ukraine), Ivan Kravtsiv (Ukraine), Janusz Stafiej (Poland), Fabio Aarão Reis (Brazil), front Dung di Caprio (France). Note that the persons in this photograph contributed to this special issue, with the exception of Ivan Kravtsiv for whom JeanPierre was a scientific grandfather, Ivan having stayed in Jean-Pierre's team during his Ukrainian-French joint $\mathrm{PhD}$.

Jean-Pierre Badiali was a very good Teacher. For some of his coworkers he was a scientific pater, for others he has been a guide in Science or a companion. But for everybody he will remain in their memory as an excellent scientist and reliable friend. J. Goodisman has words of his collaboration with Jean-Pierre which echo many colleagues' feelings: an excellent and perspicacious scientific mind and also on a personal level is Jean-Pierre's "calme et douceur" (calm and sweetness) and his care in never offending, or saying a bad word about anyone. He was a remarkably lovely person. Everyone he encountered here was charmed by his personality and impressed by his science. He was in the strongest and most literal sense a real gentle man (un homme vraiment noble).

In figure 2, celebration of Jean-Pierre's birthday in his laboratory in 2013.

The papers published in this issue were prepared by the previous PhD students, colleagues and friends of Jean-Pierre. We express our gratitude to all of them.

Dung di Caprio (Chimie ParisTech, PSL Research University, CNRS, Institut de Recherche de Chimie Paris (IRCP), Paris, France)

Wei Dong (Ecole Normale Supérieure de Lyon, Laboratoire de Chimie, CNRS, Lyon, France)

Douglas Henderson (Department of Chemistry and Biochemistry Brigham Young University, Provo,

Myroslav Holovko (Institute for Condensed Matter Physics of the National Academy of Sciences of Ukraine, Lviv, Ukraine)

\section{References}

1. Cachet H., Condens. Matter Phys., 2017, 20, 33701, doi 10.5488/CMP.20.33701.

2. Gujt J., Dominguez H., Sokolowski S., Pizio O., Condens. Matter Phys., 2017, 20, 33603, doi $10.5488 /$ CMP.20.33603

3. Regnaut C., Badiali J.P., Dupont M., Phys. Lett. A, 1979, 74, 245, doi 10.1016/0375-9601(79)90783-7

4. Regnaut C., Amokrane S., Condens. Matter Phys., 2017, 20, 33702, doi 10.5488/CMP.20.33702. 
5. Badiali J.P., Goodisman J., J. Phys. Chem., 1975, 79, 223-232, doi $10.1021 /$ j100570a007

6. Badiali J.P., Goodisman J., J. Electroanal. Chem. Interfacial Electrochem., 1975, 65, 523, doi $10.1016 / 0368-1874(75) 85141-0$.

7. Badiali J.P., Goodisman J., J. Electroanal. Chem. Interfacial Electrochem., 1978, 91, 151, doi $10.1016 / \mathrm{S} 0022-0728(78) 80097-7$

8. Amokrane S., Badiali J.P., J. Electroanal. Chem. Interfacial Electrochem., 1989, 266, 21, doi 10.1016/0022-0728(89)80212-8.

9. Zhang Q., Badiali J.P., Su W.H., J. Chem. Phys., 1990, 92, 4609, doi:10.1063/1.457722

10. Zhang Q., Badiali J.P., Rosinberg M.L., J. Mol. Liq., 1991, 48, 123, doi 10.1016/0167-7322(91)80004-N

11. Badiali J.P., Forstmann F., Chem. Phys., 1990, 141, 63, doi:10.1016/0301-0104(90)80019-T

12. Badiali J.P., J. Chem. Phys., 1989, 90, 4401, doi $10.1063 / 1.456626$

13. Zhang Q., Badiali J.P., Mol. Phys., 1992, 75, 325, doi $: 10.1080 / 00268979200100261$

14. Levesque D., Condens. Matter Phys., 2017, 20, 33601, doi:10.5488/CMP.20.33601.

15. Russier V., Ngo E., Condens. Matter Phys., 2017, 20, 33703, doi/10.5488/CMP.20.33703

16. Zhang Q., Badiali J.P., Phys. Rev. Lett., 1991, 67, 1598, doi $10.1103 /$ PhysRevLett.67.1598

17. Zhang Q., Badiali J.P., Phys. Rev. A, 1992, 45, 8666, doi 10.1103/PhysRevA.45.8666

18. Di Caprio D., Zhang Q., Badiali J.P., Phys. Rev. E, 1996, 53, 2320, doi:10.1103/PhysRevE.53.2320

19. Di Caprio D., Badiali J.P., Russier V., J. Stat. Phys., 1995, 80, 1241, doi $10.1007 /$ BF02179870.

20. Yukhnovskii I.R., Holovko M.F., Statistical Theory of Classical Equilibrium Systems, Naukova dumka, Kyiv, 1980 (in Russian).

21. Stafiej J., Badiali J.P., J. Chem. Phys., 1997, 106, 8579, doi $10.1063 / 1.473919$

22. Di Caprio D., Stafiej J., Badiali J.P., Electrochim. Acta, 2003, 48, 2967, doi:10.1016/S0013-4686(03)00362-1

23. Di Caprio D., Stafiej J., Badiali J.P., J. Chem. Phys., 1998, 108, 8572, doi $10.1063 / 1.476286$

24. Di Caprio D., Badiali J.P., J. Phys. A: Math. Theor., 2008, 41, 125401, doi: $10.1088 / 1751-8113 / 41 / 12 / 125401$.

25. Frusawa H., Hayakawa R., Phys. Rev. E, 1999, 60, R5048, doi:10.1103/PhysRevE.60.R5048

26. Patsahan O., Mryglod I., Condens. Matter Phys., 2012, 15, 24001, doi:10.5488/CMP.15.24001.

27. Di Caprio D., Stafiej J., Badiali J.P., Mol. Phys., 2003, 101, 2545, doi $10.1080 / 0026897031000154293$

28. Di Caprio D., Badiali J.P., Holovko M., J. Phys. A: Math. Theor., 2009, 42, 214038, doi $10.1088 / 1751-8113 / 42 / 21 / 214038$

29. Sergiievskyi V., Levesque M., Rotenberg B., Borgis D., Condens. Matter Phys., 2017, 20, 33005, doi $10.5488 /$ CMP.20.33005

30. Stafiej J., di Caprio D., Badiali J.P., Phys. Rev. E, 2000, 61, 3877, doi 10.1103/PhysRevE.61.3877

31. Badiali J.P., Blum L., Rosinberg M.L., Chem. Phys. Lett., 1986, 129, 149, doi 10.1016/0009-2614(86)80187-7

32. Holovko M., Vakarin E.V., Duda Yu.Ya., Chem. Phys. Lett., 1995, 233, 420, doi:10.1016/0009-2614(94)01480-J

33. Holovko M.F., Vakarin E.V., Mol. Phys., 1996, 87, 1375, doi 10.1080/00268979600100931

34. Vakarin E., Duda Yu., Holovko M., J. Chem. Phys., 1997, 107, 5569, doi:10.1063/1.474233

35. Voronov A., Luzinov J., Minko S., Sidorenko A., Vakarin E., Holovko M., Macromolecules, 1997, 30, 6929, doi $10.1021 / \mathrm{ma} 9704440$.

36. Vakarin E.V., Badiali J.P., J. Phys. Chem. B, 2006, 110, 18074, doi $10.1021 /$ jp063353e.

37. Vakarin E.V., Filippov A.E., Badiali J.P., Phys. Rev. Lett., 1998, 81, 3904, doi 10.1103/PhysRevLett.81.3904.

38. Vakarin E.V., Filippov A.E., Badiali J.P., Holovko M.F., Phys. Rev. E, 1999, 60, 660, doi $10.1103 /$ PhysRevE.60.660.

39. Vakarin E.V., Filippov A.E., Badiali J.P., Surf. Sci., 1999, 422, No. 1-3, L200, doi $10.1016 /$ S0039-6028(98)00900-5

40. Vakarin E.V., Badiali J.P., Holovko M.F., Chem. Phys. Lett., 2002, 359, No. 3-4, 349, doi 10.1016/S0009-2614(02)00723-6.

41. Vakarin E.V., Badiali J.P., Surf. Sci., 2002, 513, No. 3, 431, doi:10.1016/S0039-6028(02)01728-4.

42. Vakarin E.V., Badiali J.P., Electrochim. Acta, 2001, 46, No. 26-27, 4151, doi $10.1016 /$ S0013-4686(01)00692-2

43. Vakarin E.V., Badiali J.P., J. Phys. Chem. B, 2002, 106, No. 32, 7721, doi $10.1021 /$ jp0209190

44. Vakarin E.V., Badiali J.P., Solid State Ionics, 2004, 171, No. 3-4, 261, doi 10.1016/j.ssi.2004.05.023

45. Vakarin E.V., Badiali J.P., Levi M.D., Aurbach D., Phys. Rev. B, 2000, 63, 014304, doi $10.1103 /$ PhysRevB.63.014304

46. Vakarin E.V., Garcia-Belmonte G., Badiali J.P., J. Chem. Phys., 2007, 126, No. 23, 234709, doi $10.1063 / 1.2743410$

47. Garcia-Belmonte G., Vakarin E.V., Bisquert J., Badiali J.P., Electrochim. Acta, 2010, 55, No. 21, 6123, doi $10.1016 /$ j.electacta.2009.08.019

48. Holovko M.F., Badiali J.P., di Caprio D., J. Chem. Phys., 2005, 123, No. 23, 234705, doi $10.1063 / 1.2137707$

49. Holovko M.F., Badiali J.P., di Caprio D., J. Chem. Phys., 2007, 127, No. 1, 014106, doi:10.1063/1.2750336 
50. Henderson D., Blum L., J. Chem. Phys., 1978, 69, 5441, doi:10.1063/1.436535

51. Henderson D., Blum L., Lebowitz J.L., J. Electroanal. Chem. Interfacial Electrochem., 1979, 102, 315, doi 10.1016/S0022-0728(79)80459-3.

52. Holovko M.F., Badiali J.P., di Caprio D., J. Chem. Phys., 2008, 128, No. 11, 117102, doi:10.1063/1.2873466

53. Pecina O., Badiali J.P., Phys. Rev. E, 1998, 58, 6041, doi 10.1103/PhysRevE.58.6041.

54. Pecina O., Badiali J.P., J. Electroanal. Chem., 1999, 475, 46, doi:10.1016/S0022-0728(99)00342-3

55. Pecina O., Badiali J.P., Phys. Rev. E, 1999, 60, 4431, doi 10.1103/PhysRevE.60.4431

56. Schmickler W., Henderson H., Condens. Matter Phys., 2017, 20, 33004, doi $10.5488 / C M P .20 .33004$

57. Bhuiyan L.B., Outhwaite C.W., Condens. Matter Phys., 2017, 20, 33801, doi:10.5488/CMP.20.33801

58. González-Tovar E., Lozada-Cassou M., Condens. Matter Phys., 2017, 20, 33604, doi 10.5488/CMP.20.33604

59. Holovko M.F., Badiali J.P., Chem. Phys. Lett., 1993, 204, 511, doi 10.1016/0009-2614(93)89195-N.

60. Cassin G., Duda Y., Holovko M.F., Badiali J.P., Pileni M.P., J. Chem. Phys., 1997, 107, No. 7, 2683, doi $10.1063 / 1.474578$

61. Di Caprio D., Vautrin-Ul C., Stafiej J., Saunier J., Chaussé A., Féron D., Badiali J.P., Corros. Sci., 2011, 53, 418-425, doi:10.1016/j.corsci.2010.09.052

62. Nainville I., Lemarchand A., Badiali J.P., Phys. Rev. E, 1996, 53, 2537, doi 10.1103/PhysRevE.53.2537

63. Lafage M., Windel D., Russier V., Badiali J.P., Electrochim. Acta, 1997, 42, 2841, doi $10.1016 / \mathrm{S} 0013-4686(97) 00105-9$

64. Taleb A., Chaussé A., Dymitrowska M., Stafiej J., Badiali J.P., J. Phys. Chem. B, 2004, 108, 952, doi $10.1021 / \mathrm{jp} 035377 \mathrm{~g}$

65. Barabas A.L., Stanley H.E., Fractal Concepts in Surface Growth, Cambridge University Press, Cambridge, 1995.

66. Saunier J., Chaussé A., Stafiej J., Badiali J.P., J. Electroanal. Chem., 2004, 563, 239, doi $10.1016 /$ j.jelechem.2003.09.017.

67. Saunier J., Dymitrowska M., Chaussé A., Stafiej J., Badiali J.P., J. Electroanal. Chem., 2005, 582, 267, doi $10.1016 /$ j.jelechem.2005.03.047.

68. Aarão Reis F.D.A., Stafiej J., Badiali J.P., J. Phys. Chem. B, 2006, 110, 17554, doi:10.1021/jp063021+

69. Aarão Reis F.D.A., Badiali J.P., Pauporté Th., Lincot D., J. Electroanal. Chem., 2006, 598, 27 , doi $10.1016 /$ j.jelechem.2006.08.008

70. Vautrin-Ul C., Taleb A., Stafiej J., Chaussé A., Badiali J.P., Electrochim. Acta, 2007, 52, 5368, doi $10.1016 /$ j.electacta.2007.02.051

71. Vautrin-Ul C., Mendy H., Taleb A., Chaussé A., Stafiej J., Badiali J.P., Corros. Sci., 2008, 50, 2149, doi $10.1016 /$ j.corsci.2008.03.012

72. Di Caprio D., Vautrin-Ul C., Stafiej J., Chaussé A., Féron D., Badiali J.P., Corros. Sci., (in press).

73. Aarão Reis F.D.A., Badiali J.P., di Caprio D., Langmuir, 2012, 28, 13034, doi:10.1021/la301840c

74. Aarão Reis F.D.A., Condens. Matter Phys., 2017, 20, 33803, doi 10.5488/CMP.20.33803.

75. Zenkri M., di Caprio D., Pérez-Brokate C., Féron D., de Lamare J., Chaussé A., Ben Cheikh Larbi F., Raouafi F., Condens. Matter Phys., 2017, 20, 33802, doi 10.5488/CMP.20.33802

76. Demaerel T., Maes C., Condens. Matter Phys., 2017, 20, 33002, doi:10.5488/CMP.20.33002.

77. Holovko M., Patsahan T., Dong W., Condens. Matter Phys., 2017, 20, 33602, doi:10.5488/CMP.20.33602

78. Taleb A., Ivanova V., Condens. Matter Phys., 2017, 20, 33804, doi 10.5488/CMP.20.33804

79. Patsahan T., Taleb A., Stafiej J., Holovko M., Badiali J.P., Condens. Matter Phys., 2017, 20, 33003, doi $10.5488 /$ CMP.20.33003

80. Vakarin E.V., Badiali J.P., Cent. Eur. J. Phys., 2004, 2, 241, doi 10.2478/BF02475630.

81. Vakarin E.V., Badiali J.P., Phys. Rev. E, 2006, 74, 036120, doi $10.1103 /$ PhysRevE.74.036120

82. Vakarin E.V., Badiali J.P., Electrochim. Acta, 2011, 56, No. 10, 3526, doi:10.1016/j.electacta.2010.09.097

83. Badiali J.P., Phys. Rev. E, 1999, 60, 2533, doi:10.1103/PhysRevE.60.2533

84. Badiali J.P., Condens. Matter Phys., 2000, 3, No. 3, 545, doi:10.5488/CMP.3.3.545

85. Badiali J.P., Condens. Matter Phys., 2003, 6, No. 3, 375, doi $10.5488 / C M P .6 .3 .375$

86. Badiali J.P., J. Phys. A: Math. Gen., 2005, 38, 2835, doi $10.1088 / 0305-4470 / 38 / 13 / 002$

87. Badiali J.P., J. Phys. A: Math. Gen., 2006, 39, 7175, doi $10.1088 / 0305-4470 / 39 / 23 / 001$

88. Badiali J.P., J. Electroanal. Chem., 2012, 676, 40, doi $10.1016 /$ j.jelechem.2012.04.024

89. Badiali J.P., Condens. Matter Phys., 2013, 16, No. 4, 43003, doi:10.5488/CMP.16.43003

90. Badiali J.P., J. Phys. Conf. Ser., 2015, 604, No. 1, 012002, doi 10.1088/1742-6596/604/1/012002

91. Badiali J.P., Condens. Matter Phys., 2005, 8, No. 4, 655, doi $10.5488 /$ CMP.8.4.655

92. Riot P., Le Méhauté A., Condens. Matter Phys., 2017, 20, 33001, doi 10.5488/CMP.20.33001 